\title{
Case Study of Inclusive Education in the Context of Kuwait: Barriers and Challenges
}

\author{
Dr. Nouf Alenezi \\ College of Basic Education/Special education department \\ Public Authority for applied education and training
}

\begin{abstract}
The issue of inclusion of disabled students in mainstream schools is seen as a key issue for educational policy-makers worldwide. The argument for the inclusion of all disabled students in mainstream school's rests on human rights, as is recognized in the 'Salamanca Statement' (UNISCO,1994). However, signatories to the Salamanca statement interpret 'inclusion' in different ways, leading to the exclusion of certain groups of students. The concept of inclusion, through a review of major issues and limitations in the current practice of inclusion is highlighted by this case study. This study found that the dominant conceptual model underpinning policy and practice in Kuwait is the "medical model" of disability. This understanding lends itself to practices of integration rather than inclusion.

A multi-method data collection approach, using semi-structured interviews with teachers and parents, and critical discourse analysis of inclusion policy, has led to identifying what has been done, so far in practice, for inclusion, as well as identifying the main barriers of inclusive education in the context of Kuwait and the potential changes that need to be made.
\end{abstract}

Key words: Inclusive Education, medical model, disability. 


\section{Introduction:}

This qualitative research inquiry explores the perspectives of a diverse range of participants, namely head teachers, teachers and mothers, towards inclusion of "slow learners" in two primary mainstream schools in the State of Kuwait. The concept of inclusion, through a review of major issues and limitations in the current practice of inclusion is highlighted by this case study. A multimethod data collection approach, using semi-structured interviews and critical discourse analysis of the policy document for inclusion in Kuwait, has led to identifying what has been done, so far in practice, for inclusion, as well as identifying the potential changes that need to be made.

Kuwait is a signatory to the UNESCO Salamanca Statement, set out in 1994, which requires states to move towards systems "enabling schools to serve all children". However, to date, "inclusion" in Kuwaiti mainstream schools is limited to two groups of children: those with Down's Syndrome and those referred to as "slow learners", a term used to describe certain children with low IQ. All other children with special educational needs are educated in segregated settings. This study examines policy and explores participants' perspectives towards the inclusion of children identified as "slow learners" in primary mainstream schools in Kuwait, in order to arrive at insights which might further the policy and practice of supporting inclusion of children with special educational needs. This study found that the dominant conceptual model underpinning policy and practice in Kuwait is the "medical model" of disability, as the current understanding and practice of inclusion, teachers' and mothers' perspectives towards inclusion and articulation of the inclusion policy in Kuwait is informed, conceptualized and affected by this model. This understanding lends itself to practices of integration rather than inclusion, However, "the term 'inclusion' replaced 'integration' and is often contrasted with 'exclusion' " (Topping \& Maloney, 2005, p.42). Inclusion means equal access and increasing the participation of students with special needs in mainstream school as promoted by the Salamanca Statement (UNESCO,1994), while integration means limited access and less participation of students with special needs in mainstream school, thus integration is "largely a 'disability' or SEN issue" (Topping \& Maloney, 2005, p.42). This study also found that perspectives of inclusion are influenced by the social, cultural and religious context of the country. 
Finally, this study concludes that in the context of Kuwait, there is a clear effect of the cultural understanding of disability on the way that the current policy of inclusive education is represented. Such cultural influence not only affects the policymakers of the region and the way inclusion is implemented, but also it effects "slow learners" in the light of how disability and inclusion are constructed by the head teachers, teachers and mothers in this study. Such cultural and social values and beliefs of Kuwaiti culture pose obstacles to the existence of inclusion in Kuwait, as promoted by the Salamanca Statement. As a signatory to the Salamanca Statement, this study suggests that in order to adequately accommodate "slow learners" and other students with disability in mainstream schools in Kuwait, policy needs to be reconceptualised.

\section{Conclusion}

Within the context of Kuwait, the negative effects of the cultural aspects that produce the notion of helplessness and dependency of individuals with disability could strongly affect the implementation of inclusive education, which is seen as suitable for some but not all students with disability. Inclusion and participation is essential to human dignity and the concept of human rights to which all children are entitled. The Salamanca Statement (UNESCO, 1994) emphasises that inclusive schooling has a major role in achieving these rights and advises governments to adopt this concept as law or policy, unless they are absolutely incapable of doing so (Kite, 2008). However, in the context of Kuwait, it is almost impossible not to associate provisions offered to learners with disabilities without considering the society's traditions, attitudes, and the current cultural convictions and beliefs about individuals with disability. Many exclusionary practices are often covert and hidden within deeply embedded cultural practices (Corbett, 1999). Moreover, the way disability is considered in Kuwait shapes inclusion practices and defines the challenges of developing inclusive schools.

In Kuwait, the current state of inclusive education may be described as having a weak inclusion policy with a particular understanding and practice of inclusion. The current practice reflects the 


\section{LENAEDULATION

integration ethos rather than the inclusion ethos. In Kuwait, it is interesting to note how the principles of inclusion - equal access - as promoted by the Salamanca Statement, translate into "excluding the included". "Slow learners" are included in mainstream schools, however in practice they are excluded from most aspects of school life activities. The use of the term "exclusion" in this present study is based on the explanation of Booth (1998, pp.34-35) who states that, "exclusion, like segregation, can be conceived of as the process of decreasing the participation of pupils in the cultures and curricula of mainstream schools". "Slow learners" are considered as outsiders in their own school, as revealed by the participants in this study. However Kearney and Kane (2006, p.205) indicate:

To be included is not to be excluded. Exclusion and inclusion are two sides of the same coin and to understand one, requires an understanding of the other.

The way inclusive education operates is ultimately not inclusive. The data demonstrate notions of exclusion paradigms, based on a special education knowledge base, by creating special classes for students who are "slow learners" in mainstream schools. This is undertaken without any planning, beyond location, to include them within mainstream school life. This displays some recognition of the part played by social and cultural forces in defining disability, which is consistent with the medical model of understanding disability. Armstrong et al. (2010, p.44) suggest that:

The way in which 'inclusion' is conceptualized may differ significantly in the practice of policy-makers, administrators, principals and teachers, and other professionals working in different national educational contexts.

Thus, it can be argued that the current understanding and practice of inclusion, teachers' and mothers' perspectives towards inclusion and articulation of the inclusion policy in Kuwait is informed and conceptualised by the local culture of the region. In the context of Kuwait, there is a clear effect of the cultural understanding of disability on the way that the current policy of inclusive education is represented. Such cultural influence not only affects the policymakers of the region and the way inclusion is implemented, but also it affects "slow learners" as to how disability and 
inclusion is constructed by teachers and mothers in this study. Hassanein (2015, pp.1-2) argues that:

Inclusion, within western cultures is seen as a universal approach to providing educational opportunities for children with 'special educational needs' in ordinary school settings. However, such an approach may act differently in other cultural contexts, many cultural backgrounds, including Egyptians, have their own long-standing beliefs and practices which do not define or address disability in the same way as western culture, such cultural differences in understanding disability may lead to different understandings, conceptualisations and practices of inclusion.

In this regard, the cultural and social values, beliefs, traditions and background of Kuwaiti culture, which does not see disability in the same way as in the west (e.g., UK; USA) could pose obstacles to the existence of inclusion in Kuwait and the development of inclusive thinking. Such cultural aspects interact and clash, creating a contradictory situation both for individuals with disability and inclusive education in Kuwait.

This discussion draws on theory and research in the connected fields of disability studies and inclusive education. I believe, that by exploring the way of understanding disability in the culture of the region could be considered as a starting point to be used as a vehicle for change towards a more inclusive education policy and practice in Kuwait. Ainscow and Sandill (2010, p.412) claim that, "the focus must not only be on practice. It must also address and sometimes challenge the thinking behind existing ways of working". This study identifies that in Kuwait, the situation not only lacks vision and seriousness but also knowledge of inclusive thinking. Consequently, the significant parts of the framework that is required for achieving inclusion are missing. The next chapter presents the contributions, implications and recommendations of this study.

\section{Contribution to knowledge}


This study has undertaken research into the concepts of disability and inclusive education in an Arab Muslim country, where research in this field is scarce. It contributes to knowledge by revealing attitudes and perspectives and relating them to prevailing policy and cultural contexts in the State of Kuwait. Its uniqueness is founded in exploring the perspectives of head teachers, teachers, and parents towards inclusion and relating these to the local policy context. In doing so it gives rise to a number of recommendations to enable Kuwait to better meet its obligations as a signatory to the Salamanca Statement. Conceptualisation of inclusion in countries in the Arabian Gulf region is limited, as such this study contributes to the understanding of inclusion through its consideration of multiple factors. It has identified cultural beliefs towards individuals with disability and explored the legislation of inclusion and the practice of inclusion. The research has also shed light on the translation of policy from global to national level and the importance of cultural contexts in this process.

This study contributes to the knowledge of inclusive education by showing how models of disability have shaped educational policy and practice offered to disabled children in general and specifically to "slow learners" in Kuwait. It also contributes to the knowledge of inclusive education by showing how models of disability have shaped educational policy and practices in different cultural contexts. This study has confirmed the dominance of the medical model of understanding disability among the participants and in the articulation of the policy document of inclusion in Kuwait. This is influenced by the culture of the region, which is consistent with the assumptions of such a model. The cultural context results in overprotective environments, promotes dependency and focuses on impairments, when viewing the needs of care for individuals with disability. Such an understanding of disability affects independence and inhibits complete inclusion in mainstream schools. Furthermore, this research contributes by enabling individuals to become critically aware of the concepts of care within the culture of the region and practices that this involves. It provide more informed understanding of care, and has presented a critical reappraisal of the construct of "care" within different model of understanding disability. 
This study contributes to knowledge by suggesting reconsideration of the way disability is understood, as well as the way of caring within the culture of Kuwait. Such reconsideration could lead to changes in current exclusive thinking and practices. Thus, this research contributes to our knowledge about inclusion within Kuwaiti culture and provides a theoretical framework about inclusion in Kuwait at different levels, policy, culture and practice.

\section{Implications}

This study gives rise to a number of implications, in the field of inclusive education. Inclusive education in Kuwait is dominated by the medical model of understanding disability and is affected by the collectivist culture that produces an overprotective care environment, especially for individuals with disability. Care is provided without limit and without encouragement to lead an independent life. In the context of Kuwaiti cultural norms, care and the resulting overprotective environment, relating to individuals with disability, results in these individuals struggling to achieve the same level of independence as their non-disabled peers. Gaad's study (2011, p.82) of inclusive education in the Middle East indicates that:

Care and concern for individuals having special needs and/or disabilities has been a component of both heritage and culture from time immemorial. Challenges lie, however, in identifying and meeting the needs of individuals and how cultural understanding, or perhaps misunderstanding, of their needs can play a role in such a caring process.

Thus, considering the "ethics of care" and the distinction between expressed and inferred needs (Noddings, 1984) could further the practice of inclusion in mainstream schools if it were to be considered by the policymakers of inclusive education in Kuwait. Brisenden (1986, pp.176-177) effectively encapsulates an ideal situation which could guide policy-makers and practitioners in this regard:

We should instead look at a life as a whole, and allow people with disabilities to take decisions for themselves based on many other factors as well as medical ones. We have to look at a person's independence and ask how this can be assisted and promoted without 


\section{LENAEDULATION \\ 27-29 March, 2020 \\ Oxford, United Kingdom}

taking the right of control away from the individual.

Noddings (2005, p.158) believes that there is more to education than just getting high grades and test scores. There is also a strong need to consider wider concerns and continual reflection that "should help us in the task of balancing expressed and inferred needs". Mitchell (2005, p.14) claims that:

Inclusive education is embedded in a series of contexts, extending from the broad society, through the local community, the family, the school and to the classroom.

Clearly, consideration of Noddings' "ethics of care", regarding the "balancing of expressed and inferred needs" and considering it as an essential element in inclusive education, could contribute to removing barriers to learning and participation of all students, as indicated in the previous chapter.

The index for inclusion states that in order to become inclusive, school communities should restructure overall school organisation, acknowledge student diversity, increase participation of all students and remove barriers to learning and participation of all students (Booth \& Ainscow, 2002). Defining inclusion as valuing diversity or "a set of principles which ensures that the student with disability is viewed as a valued and needed member of the community" (Uditsky, 1993, p.88), is not enough. Barton (1998, pp.84-85) stresses that, "inclusive education is about participation of all children and young people and the removal of all forms of exclusionary practice". Inclusive education should not just be about participation and removal of the exclusionary practices, it should be also about listening, being open and empowering everyone regardless of ability or disability. Barton (1998, pp.84-85) believes that successful inclusive education should be "about learning to live with one another". Florian, 2014, p.293) suggests that:

What counts as evidence of inclusive education is an important question that can be partially answered by an approach to the study of teachers' practices that specifies principles, assumptions and actions.

The most important issue is to theorise practice and attempt to recognise the actual needs of 
students with disability, in inclusive schools, in order to overcome the criticism of the concept of inclusive education that has been described as "promising more than it delivers" (Florian, 2014, p.286). In the UK, Ainscow (1991) links inclusive education to ideas of school improvement, arguing for the focus of special education to shift away from differences between learners towards changing school practices. In the context of Kuwait, this study suggests that to put Noddings' perspectives of "balancing expressed and inferred needs" at the heart of the policy of inclusive education would be a step towards changing current practices of inclusive education. Noddings' view is that, from the perspective of "ethics of care", the objective should be to understand what the cared-for is experiencing and to hear and understand their expressed needs. Noddings (2012, p.772) asserts that:

The teacher as carer is interested in the expressed needs of the cared-for, not simply the needs assumed by the school as an institution and the curriculum as a prescribed course of study.

Applying Noddings' views should firmly place the student with disability at the centre of any policy and/or practice developments of inclusive education in Kuwait. Noddings (2012) highlights very significant aspects that could be used to theorise the practice of inclusion and achieve its fundamental aims, such as participation and responding to diversity. "Instead of meeting the expressed need, the carer's objective is to maintain the caring relationship" (Noddings, 2012, p.772). However, this may not always be possible for a range of reasons, including lack of resources or disapproval of the need, or the way it may be expressed. In such a case, it is essential to respond in a way that keeps the door of communication open, therefore the response of the cared-for completes the caring relationship (Noddings, 2012). This new thinking, based on the "ethics of care" theory, implies and creates a new role, in the context of Kuwait, for services for people with disabilities and for the people engaged with them. Such a way of responding towards the cared-for - the disabled child - means achieving his/her well being and valuing diversity in inclusive schools; the existence of these two principles is key in the field of inclusive education. 
Legislation is seen as playing a crucial part in the efforts towards inclusive education (UNESCO, 1994). This is the first study in Kuwait to apply the CDA approach to analysing policy, with the aim to identify the gaps and provide a reference point for the government as a better future guide, where "policy is decisively shaped by powerful structural forces of an economic, ideological and cultural nature" (Bell \& Stevenson, 2006, p. 9). As such, CDA has helped to reveal the nature of these ideological and cultural forces and reveal the power relations among them. In addition, the CDA approach, applied to the policy document, has helped to identify the effect of culture on the articulation of policy. It confirms the absence of equal rights discourse in the document, and highlights a disparity with the Islamic religion. Hassanein (2015, p.174) provides a practical example of how Islam respects human beings and their differences:

One of the religious experiences that might have supported positive attitudes could be the social contact with disabled people in the five daily prayers in the mosque. In the mosque you are supposed to see all kinds of human beings; different colours, different abilities, different languages, etc. Additionally, the dilemma of difference is simply addressed in Islam as a feature of the human nature with nothing to do with the human dignity which is reserved for every single human being.

The absence of equal rights discourse is an effect of the culture and is consistent with the medical model assumption, which distinguishes between able and disabled, normal and abnormal and encourages the notion of difference. The findings of this study support this point as well as the effect of the culture on the way of understanding the principles of Islam, as discussed previously. Therefore, this implies the need for a religious discourse on inclusion and disability to explore the role of religious values and other cultural notions and misconceptions in shaping policy and participants' views of inclusion and other controversial issues. Florian (2008) maintains that inclusive education accepts differences between students as normal aspects of human development. Current educational policies and practices, in Kuwait, should seriously consider the Islamic moral system in inclusive educational practices. 


\section{SICNAEDULATION \\ 27-29 March, 2020 \\ Oxford, United Kingdom}

\section{Recommendations}

The following points are vital aspects which need to be considered in an attempt to draw the attention of policymakers, practitioners, researchers and stakeholders towards improving inclusive education in Kuwait.

\section{Promoting equal rights discourse for all students with disability in policy and practice}

The concept of widening participation in mainstream schools, as a means of ensuring quality education for all learners, is one of the key principles of inclusion as indicated in the Salamanca Statement (UNESCO, 1994) to which Kuwait is a signatory. Cookson (1994, p.119) suggests that, "The significance of the power discourse at the national legislative level cannot be underestimated". However, this study has identified that the equal rights discourse does not exist in the inclusion policy in Kuwait, and such a gap in the policy strongly affects the rights of "slow learners" in mainstream schools.

Re-articulating the policy of inclusion in Kuwait is a key step towards improving inclusion. Rearticulation would need to consider the Salamanca Statement's principles to promote and encourage the protection of the rights of students with disability in inclusive schools, meet learners' diversity, and emphasise their right to equal participation. Fidler (1996, p.2) considers that, "Wellness and well-being are a result of participation in personally and socially relevant activities that focus on and maximise individual strengths and capacities". Thus, promoting equal rights for "slow learners" in mainstream schools, with an aim to achieve their wellbeing, should be at the heart of inclusive education policy in Kuwait.

\section{Understanding disability through the lens of the social model}

"Understanding the causes of disability opens more space for understanding the inclusion process" (Šiška \& Habib, 2013, p.403). The current study identifies that the dominance of the medical model 


\section{LENAEDULATION

of understanding disability affects the articulation of the policy document of inclusion, as well as most of the participants' perspectives towards inclusion, which in turn affects the practice of inclusion in Kuwait. In this regard, first the content of the current policy of inclusion should be changed by redefining disability and the concept of inclusion. It should frame disability and inclusion under the umbrella of the social model of understanding disability, which is human rights-based, rather than the umbrella of the medical model of understanding disability. Doing so could contribute to improving the practice of inclusion in Kuwait. Oliver and Barnes (1996, p.66) explain the aim of developing the social model by emphasising that:

The social model was developed to counter the formidable tragedy discourse that surrounds disabled people and that depicts disability as a deficit, a tragedy and abnormal, and something to be avoided at all cost.

Secondly, the socio-cultural context, which depicts disability as a "problem that has to be fixed", needs to change because human beings are social creatures who have the need to live within communities and socialise with others. It is important to distinguish between the terms "impairment" which is an individual organic dysfunction and "disability" which is restriction in response to the needs of individuals with disability (UPAIS, 1976 cited in Oliver, 1996). It is also important to overcome the deficit orientation to difference, which is consistent with the medical model that remains so influential among the participants in this study. Thus, increasing awareness and the level of training for pre-service and in-service teachers, regarding their way of viewing and understanding disability, should be in the heart of any education policy.

\section{Increasing awareness about the concept of inclusion}

The findings indicate that among the majority of the participants there is limited understanding of inclusion, that involves only physical movement from mainstream classes to special classes and participation in some but not all mainstream school life. Such an understanding reflects integration rather than inclusion. Furthermore, such understanding of inclusion is also reflected by the policy of inclusion in Kuwait as indicated in Chapter Four. It is important that the policy of inclusion in Kuwait addresses the main principles of inclusion, as well as defining the concept of inclusion 


\section{SICNAEDULATION

presented in the Salamanca Statement, which is linked to acknowledgement that, "quality education for learners with SEN in mainstream schools must mean quality education for all learners" (European Agency for Development in Special Needs Education, 2009, p.9). Such a policy needs to explain that for mainstream schools to be inclusive they need to consider:

The challenge confronting the inclusive school is that of developing a child-centred pedagogy capable of successfully educating all children, including those who have serious disadvantages and disabilities. The merit of such schools is not only that they are capable of providing quality education to all children; their establishment is a crucial step in helping to change discriminatory attitudes, increasing welcoming communities and in developing an inclusive society. (UNESCO, 1994, pp.6-7)

Thus, the concept of inclusion needs to be re-thought, with assumptions that differ from those of special education. Kuwait needs to move beyond the medical model of understanding disability and frame inclusion through the Salamanca Statement principles, as "the progress towards inclusive education is often portrayed as a matter of identifying and removing barriers to participation and learning" (Booth \& Ainscow, 2011, p.5).

\section{Implementing inclusion for other students with disability in Kuwait}

The data indicate that only students who are "slow learners" and Down's Syndrome are included in mainstream schools, while other students with disability such as students with physical disability are still excluded in special schools. However, this is in contrast with the concept of inclusive education, as presented in the Salamanca Statement, which clearly indicates that: "Education for All effectively means FOR ALL" (UNESCO, 1994, p.iv). There is no clear reason, in the current policy document about implementing inclusion for only two categories of students with disability. The State of Kuwait, being a signatory to the Salamanca Statement (UNESCO, 1994), declared its commitment to inclusive education, therefore the key question for the policymakers is: Why is inclusion implemented only for "slow learners" and Down's Syndrome students? It is important to encourage inclusive thinking in policy and practice to promote the inclusion of all students, 


\section{LENAEDULATION \\ 27-29 March, 2020 \\ Oxford, United Kingdom}

regardless of their condition, to overcome current exclusive thinking.

\section{Encouraging independence of life for individuals with disability in Kuwait}

The culture of the region, which is consistent with the medical model of understanding disability, promotes the notion of care, dependency and helplessness for individuals with disability. It also inhibits inclusion because it emphasises the notion of difference and normalcy, by offering ways of care without limits, which can stifle independence. Brisenden (1989, p.9) considers that:

Independence is not linked to the physical or intellectual capacity to care for oneself without assistance; independence is created by having assistance when and how one requires it.

Such cultural aspects of the region are in sharp contrast with the concept of inclusion, because they favour protection and care over independence. If independence is considered subordinate, then diversity may not be respected and appreciated. Coulston and Smith (2013) argue that inclusion incorporates best practice and shared values through which it supports the diversity of students. Brown (1997, p.262) claims that:

Settings that are barrier free allow people with disabilities to move about independently and in so doing, foster a sense of personal control. This perception of control is an important factor in preventing feelings of helplessness, both real and learned.

Consequently, increasing awareness among people in the region could contribute to moving towards a more inclusive society and independent life for people with disability. As an outcome of this study, key groups such as pre-service teachers, in-service teachers, non-disabled students and parents need to be educated as to how they deal with individuals with disability by responding to their "expressed needs" (Noddings, 2005) and overcoming the notion of helplessness and the provision of overprotective environments. Overemphasising individual disabilities can jeopardise attempts to develop inclusive education (Ware, 2003). However, it would be naïve to ignore the fact that such students might require medical care and support (Barton, 1993). 


\section{SICNAEDULATION \\ 27-29 March, 2020 \\ Oxford, United Kingdom}

\section{Parental involvement}

Leyser and Kirk (2004) state that parental involvement and support is critical for the successful implementation of inclusion. However, as the findings of this study show, the role of parents in developing inclusive education in Kuwait is not considered by policymakers or the Ministry of Education. On the other hand, the Salamanca Statement (UNESCO, 1994, pp.37-38) encourages and emphasises the importance of parent partnership in the success of inclusive education, indicating that:

The education of children with special educational needs is a shared task of parents and professionals. A positive attitude on the part of parents favours school and social integration. Parents need support in order to assume the role of a parent of a child with special needs. The role of families and parents could be enhanced by the provision of necessary information in simple and clear language; addressing the needs for information and training in parenting skills is a particularly important task in cultural environments where there is little tradition of schooling. Both parents and teachers may need support and encouragement in learning to work together as equal partners.

A cooperative and supportive partnership, between schools and parents, is a key element in delivering inclusive education. As Brown observes, "In countries with long-established traditions of inclusive practice, we find a considerable emphasis on professional team planning and systems for problem solving" (Brown, 2005, p.275). Thus, Kuwait needs to show its commitment to inclusion. It is time that educational policies take full account of the role of parents in the development of inclusive education, by considering their voices and views, as well as cooperating with them and supporting them.

\section{Restructure mainstream schools for inclusive education}

The way of understanding disability, from the view of the medical model in the articulation of the policy and among most of the participants in this study, affects the way of understanding and 


\section{SENAEDULATION

implementing inclusion. Inclusion is clearly seen as accommodating children with disability in an education system that is unchanged at the structural, organisation, and curriculum level. However, inclusive education is not based on a child-by-child approach, it should be undertaken as a systemwide approach (Miles, 1999). Therefore, existing school systems and curricula, in inclusive schools in Kuwait, should be redesigned in a more comprehensive and extensive way by adopting the Salamanca Statement's framework. This provides clear and detailed guidance of the key school factors that should exist in inclusive schools, such as curriculum flexibility indicating that in inclusive schools:

Curricula should be adapted to children's needs, not vice versa. Schools should therefore provide curricular opportunities to suit children with different abilities and interests. (UNESCO, 1994, p.22)

Restructuring the curriculum would be a step forward, however without associated organisational change the effectiveness of curricular changes will be reduced.

\section{Ensuring all teachers are trained and feel able to assume responsibility for all learners, whatever their individual needs}

The preparation of teachers for inclusive education requires suitable training to be available both during initial teacher education courses and for practicing teachers (Sharma et al., 2012a). The findings from this study, show that teachers point to a lack of training opportunities about inclusion and students with disability, in both pre-service and in-service training. This study has identified some important practical implications for teacher education in Kuwait. All who work in schools should be educated to overcome their negative views of difference, which may be deeply entrenched and which could define certain categories of students as lacking something (Trent et al., 1998). In addition, teachers' understanding and knowledge about the principles of inclusion should be increased in order to enable them to identify their role in promoting greater inclusion practices. They should be aware that, as Slee (2007) indicates, inclusive education is not the 


\section{SICNAEDULATION

adaptation or refinement of special education, it is a complete rejection of the claims of special education and regular education to be inclusive. Symeonidou and Phtiaka (2009) note that teachers with university preparation have more positive attitudes towards inclusion and greater self-esteem when covering the educational needs of children with disabilities. As the findings of the current study suggest, teachers lack both the necessary knowledge and attitudes in order to support inclusive education. Therefore, improving the level of training for teachers regarding inclusion and learner diversity is one of the essential factors that could contribute to the development of inclusive education in Kuwait.

\section{Engendering teamwork in inclusive schools}

This study identified a lack of cooperation between mainstream and special teachers in inclusive schools. Some mainstream teachers are prevented, by the inspector of the special classes, from engaging with, visiting or working with the special teachers. Such practices reflect current exclusive thinking and are in sharp contrast with the principles of inclusion, which require cooperation and communication among teachers, with an aim to meet all students' needs and diversity. Therefore, the policy of inclusion should emphasise this fundamental factor and encourage inspectors, teachers, parents and all school staff to work as a team to achieve inclusion, as indicated by the Salamanca Statement:

Each school should be a community collectively accountable for the success or failure of every student. The educational team, rather than the individual teacher, should share the responsibility for the education of special needs children. Parents and volunteers should be invited to take an active part in the work of the school. (UNESCO,1994, p.24)

\section{Teachers, head teachers and parents must be aware of the content of the policy}

The data indicate that none of the participants in the current study are aware of the content of the policy Regulation of law 4 of 1996, as the Ministry of Education plays no role in increasing the awareness of schools about the policy content. The fact is that the current policy includes several 


\section{LICNAEDUCATIION}

limitations, related to inclusive education, which are not very helpful for schools. However, for future purposes it is important that all stakeholders can access the policy and indeed, contribute to its development as well as its implementations. Green and Shinn (1995) suggest that sufficient information about the benefits of inclusion is likely to have a positive effect on attitudes towards inclusion. Thus, it is recommended that the policy of inclusion in Kuwait is published electronically and made widely available, with the aim for it to be a reference for teachers, head teachers and parents, as needed.

\section{Practical recommendations and suggestions}

Based on the findings of this study, the following recommendations and suggestions are of significant value and contribute to the development of the current practice of inclusion in Kuwait.

\section{Recommendation A}

The process of inclusion in Kuwait needs support and direction from previous studies about the required educational context and planning.

\section{Suggestions}

- to provide practical courses and visits to other countries, who have had successful experiences with inclusive education, for the acquisition of new information about the development of inclusive education;

- to encourage researchers to conduct research and surveys about inclusion and disability.

\section{Recommendation B}

The current implementation of inclusion is undertaken without any planning or organisation, therefore the concept of inclusion should be fully understood by those in education before any implementation. 


\section{LICNAEDUCATIION \\ 27-29 March, 2020 \\ Oxford, United Kingdom}

\section{Suggestions}

- the Ministry of Education should design clear plans and clarify for schools, parents, teachers and students the concept of inclusion, before its implementation;

- special committees should be established to assess teachers, before transferring them to teach students who are "slow learners";

- the teacher training curriculum should include awareness lessons about inclusion and students

with disability.

\section{Recommendation $\mathbf{C}$}

Increase the financial support for inclusive schools.

\section{Suggestions}

- prepare school buildings to be suitable for all student diversity;

- suitably equip and resource classes, with electronic (e.g. data show or smart boards) and standard facilities.

\section{Recommendation D}

To raise pupils' respect for each other to reduced levels of bullying and peer exclusion.

\section{Suggestions}

- before implementing inclusion, non-disabled students need to be educated about students who are "slow learners" and how to deal with them and respect them, in order to avoid bullying;

- the participation of students who are "slow learners" in all school activities, not only in some subjects, needs to be increased.

\section{Recommendation $\mathbf{E}$}

For successful inclusion the awareness and the knowledge of head teachers, teachers, other school staff, parents, and students about the concept of inclusion and disability need to be developed. 


\section{LICNAEDUCATIION \\ 27-29 March, 2020 \\ Oxford, United Kingdom}

\section{Suggestions}

head teachers

- train head teachers and increase their knowledge about inclusion and students with disability;

teachers

- develop specialists in the field of inclusion, who understand the situation of students who are "slow learners" and their needs ;

- provide teacher training courses regularly to explain the aim and concept of inclusion and how to deal with students who are "slow learners;

other school staff

- the school administration and all staff of the school should also be prepared for the inclusion of students with disability, not only the teachers;

parents

- organise seminars or training courses for parents of non-disabled students and parent of students who are "slow learners", in order to increase their awareness about inclusion; and how to deal with students with disability;

- establish communication links between the parents of both non-disabled students and students who are "slow learners";

\section{Recommendation $\mathbf{F}$}

Increase awareness and support for inclusion to help parents, teachers, schools and society to understand inclusion.

\section{Suggestions}

- publicise inclusion through the media;

- publish guides to inclusion for parents, schools, students and teachers;

- develop a programme to encourage families to play a role in educating and increasing the awareness of their children about how to deal with disabled people positively.

Finally, as the findings show that there is a dominance of the medical model of understanding 
disability in the culture of the region, understanding disability through the lens of the social model and the capability model will increase awareness and change the existing understanding of disability.

\section{Strengths and limitations of the study}

Like any research enquiry, this study has its strengths and limitations. This study contributes to the further understanding of inclusive education in Kuwait. The methodological contributions arise from the case study approach, which made it possible to examine the complexities of inclusive education in Kuwait. The application of qualitative methods provided greater insight than a quantitative approach into the influences of cultural and religious factors on global and transferred policies relating to disability and inclusive education. Applying qualitative research methods provided rich sources of data for understanding inclusive education. In addition, the qualitative data analysis provided me with rich data and understanding of participants' views towards disability and inclusion, despite the small number of participants. It is impossible to obtain such a deep understanding by using quantitative methods only, because of the limited possibilities for participants to express their extended views. The use of a semi-structured open question approach, enabled me to conduct a wide-ranging exploration of ideas. Such an approach well suited the research purposes of identifying issues related to inclusive education in Kuwait.

The semi-structured interviews allowed the voices and perspectives of the different participants to be heard, in particular mothers of "slow learner" children. Sharing the thoughts, opinions, experiences and concerns of head teachers, teachers and mothers of "slow learners", as well as mothers of non-disabled students, provided a wide ranging exploration of ideas. This approach suited the research purposes, as it investigated the whole inclusion process and consequently has been able to inform stakeholders and decision-makers about making mainstream schools more inclusive for all learners.

To my knowledge, the current study is the first study to use CDA to analyse the policy document of inclusive education in the State of Kuwait. Therefore, it has value because it has started a process 
that begins to challenge the existing situation in Kuwait. Such an approach has helped to identify the contradictions and key limitations in the policy, that in turn affects the practices discussed in previous chapters. Riddell and Weedon (2014, p.364) state that it is, "very important to examine not only policy discourses but also their consequences". The current study has identified the consequences of the policy discourses, by using semi-structured interviews with teachers, head teachers and mothers to more closely identify the negative consequences of these policy discourses. This was achieved by considering the participants' concerns about the practice of inclusion in mainstream schools, and linking that with the limitations and the gaps, identified by using the CDA approach, in the Kuwaiti policy document of inclusion. Consequences identified in this study include the marginalisation of the equal rights discourses and its negative effect on the level of "slow learners" participation in mainstream schools.

Regarding the limitations of this study because of the limited time available it was conducted only in two inclusive schools in Kuwait and with a small number of participants. The value of a small scale study is supported by Flyvbjerg (2006, p.288), who corrects the misunderstanding surrounding case study, arguing that:

One can often generalize on the basis of a single case, and the case study may be central to scientific development via generalization as supplement or alternative to other methods.

My intention was not to make any generalisation across the schools, but rather to enhance an in depth understanding and gain a clearer picture of the situation of inclusive education in Kuwait. A further limitation of this study, due to cultural restriction issues, is that it did not include male teachers' and fathers' perspectives towards inclusion. This action was taken to avoid any problems that would arise for me as the researcher, in collecting data from male teachers or fathers. Furthermore, I decided to limit the scope of the current study by only considering the voices of head teachers, teachers and mothers, however even though the voices of disabled students were not heard, does not mean that such voices are not important. My reflections on undertaking the research process are included in Appendix 12. 


\section{Direction for future research}

This study only investigated the perspectives of female head teachers, teachers and mothers towards inclusion. Thus, it is important to investigate the views of male teachers and head teachers, as well as fathers, towards inclusive education. Further, this study only focused on one group of children, who are currently included in mainstream schools in Kuwait - "slow learners". Thus, it would be interesting to investigate teachers' and parents' perspectives towards including all categories of children with special education needs. Furthermore, it would be significant to investigate, in future research, the perspectives of disabled students towards inclusion.

The Salamanca Statement and framework for action recommends and advises the signatory countries that: "Pilot experiments and in-depth studies should also be launched to assist in decision-making and in guiding future action" (UNESCO, 1994, p.25). Increasing research in the field of inclusive education and understanding disability is a key factor in developing education for all in Kuwait; more studies are needed to expand this knowledge base as research in the region, in this field, is scarce.

\section{Final thoughts}

This study views inclusive education as a continuous process moving towards achieving social justice and an equal society. The barriers identified should be seen as obstacles to be overcome and not considered as discouraging the move towards inclusive education in Kuwait. This investigation should be used to clarify current practices of inclusive education in Kuwait and to provide directions for formulating policies to support inclusive practice in ways which are satisfactory to, head teachers, teachers, parents and students alike.

The aim of this study is to make stakeholders in the field of education, and the government of Kuwait, reconsider their approach to inclusive education and the way they work at present. I believe that this in-depth case study should be used as guidance for the Ministry of Education and other 
stakeholders in future action towards developing inclusive education. All stakeholders involved in education, in the State of Kuwait should consider the words of O'Toole and McConkey (1995, p.5):

"All of us need to look to the distant horizon to ensure that we are travelling on the right path". 